\section{BIBLIOGRAFÍA}

1.- COFFEY T J, DOWSON C G, DANIELS M, SPRATT B G. Genetics and molecular biology of $\beta$-lactam-resistant pneumococci. Microb Drug Resist 1995; 1: 29-34.

2.- COFFEY T J, DANIELS M, ENRIGHT M C, SPRATT B G. Serotype 14 variants of the Spanish penicillinresistant serotype $9 \mathrm{~V}$ clone of $S$. pneumoniae arose by large recombinational replacements of the cpsA-pbpla region. Microbiol 1999; 145: 2023-31.

3.- ENRIGHT MC, SPRATT B G. A multilocus sequence typing scheme for $S$. pneumoniae: identification of clones associated with serious invasive disease. Microbiol 1998; 144: 3049-60.

4.- MUSSER J M. Molecular population genetic analysis of emerged bacterial pathogens: selected insights. Emerg Infect Dis 1996; 2: 1-17.

5.- TOMASZ A, CORSO A, and the members of the PAHO/ Rockefeller University Workshop: SEVERINA E P, ECHANIZ-AVILES G, DE CUNTO BRANDILEONE M C, CAMOU T, CASTAÑEDA E, FIGUEROA O, ROSSI A DI FABIO J L. Molecular epidemiologic characterization of penicillin-resistant Streptococcus pneumoniae invasive pediatric isolates recovered in six Latin American countries: an overview. Microb Drug Resist 1998; 4: 195-207.

Correspondencia a:

María Hortal

Mail: mhortal@st.com.uy

\title{
Proyecciones de las vacunas anti neumocóccicas conjugadas en Latinoamérica
}

\author{
PERSPECTIVES FOR PNEUMOCOCCAL CONJUGATE \\ VACCINES IN LATIN AMERICA
}

\author{
EDUARDO L. LÓPEZ
}

\section{INTRODUCCIÓN}

Streptococcus pneumoniae (neumococo) es una de las causas mas frecuentes de enfermedad grave en pacientes pediátricos. Así es considerado como el agente bacteriano que provoca el mayor numero de neumonías en niños, se estima que alrededor del $30 \%$ de las neumonías son causadas por este microorganismo ${ }^{1}$. Por otra parte es una de las causas principales de meningitis bacteriana aguda (MBA) en América Latina, estimándose que se producen alrededor de 9.000 casos por año en América Latina y el área del Caribe ${ }^{2}$.

En Argentina el neumococo es el segundo agente causal de MBA según datos oficiales como se observa en la Tabla 1. Como puede observarse en dicha tabla S. pneumoniae provocó 1.318 casos de esta patología en el período de 1997 al 2000, representando $25,3 \%$ del total de casos de las MBA con aislamiento etiológico. La mortalidad de las meningi- tis provocadas por este agente es entre 20 y $25 \%$ según los centros y un tercio de los niños quedan con secuelas severas ${ }^{3}$. Debe además recordarse que en Argentina hay un subregistro de alrededor del $30 \%$. Por lo tanto se podría considerar que fallece 1,7 niños por semana por esta patología. Si aceptamos esta cifra de mortalidad como un promedio para América Latina y asumiendo los datos publicados por H. Peltola, se podría decir que fallecen semanalmente 47 niños a causa de meningitis neumocóccica.

Además $S$. pneumoniae es el agente causal de la mayoría de los cuadros de bacteremia oculta en pacientes pediátricos. Estudios efectuados en Chile por R. Lagos et al han demostrado que en niños febriles, a quienes se les efectuaba hemocultivos de rutina, en un número significativo de los mismos se aislaba neumococo ${ }^{4}$.

También S. pneumoniae provoca un número considerable de neumonías en la niñez. Estudios efectuados en Europa y en E.U.A.. (Dallas) han de-

${ }^{1}$ Hospital de Niños Dr. Ricardo Gutiérrez y Facultad de Medicina, Universidad del Salvador. Buenos Aires, Argentina. 
Tabla 1: Etiología de las meningitis bacterianas agudas en Argentina ${ }^{1}$

Año

\begin{tabular}{lrrrr}
\hline MICROORGANISMO & 1997 & 1998 & 1999 & $2000^{2}$ \\
\hline & $n$ & $n$ & $n$ & $n$ \\
N. meningitidis & 1.047 & 734 & 593 & 451 \\
H. influenzae b & 361 & 149 & 60 & 30 \\
S. pneumoniae & 404 & 303 & 328 & 283 \\
Otras etiologías & 179 & 119 & 78 & 82 \\
TOTAL & 1.991 & 1.305 & 1.059 & 846 \\
\hline
\end{tabular}

${ }^{1}$ Dirección de Epidemiología / Ministerio de Salud Argentina

${ }^{2}$ Boletín Epidemiológico 21/12/2000

mostrado que en $28 \%$ de los aislamientos en neumonías de la comunidad en niños se identificó $S$. pneumoniae $^{4}$. En el Hospital de Niño Ricardo Gutiérrez de Buenos Aires, Argentina, se internan por año entre 33 y 40 pacientes con neumonía neumocóccica incluyendo aquellas que presentan supuración pleural. En nuestra institución es el primer agente bacteriano de las neumonías que requieren internación (Vázquez y López, comunicación personal).

Finalmente se debe recordar que la otitis media aguda es la infección bacteriana más frecuente en Pediatría ambulatoria, causando el neumococo aproximadamente el $40 \%$ de las mismas ${ }^{5}$. Obviamente, si bien se trata de una afección benigna, el impacto en términos de días de enfermedad, número de afectados y costos en uso de antimicrobianos, es muy importante.

\section{Serotipos de Streptococcus pneumoniae aislados en Latinoamérica}

Uno de los aspectos más importantes a tener en cuenta para definir la utilidad de una vacuna conjugada anti neumocóccica en Pediatría es conocer los serotipos de $S$. pneumoniae de mayor prevalencia en la comunidad.

En América Latina, Kertesz et al publicaron el primer estudio multinacional sobre infecciones invasoras provocadas por $S$. pneumoniae. Los países participantes fueron Argentina, Brasil, Chile, Colombia, México y Uruguay. Se estudiaron 1.621 pacientes con menos de cinco años de edad, correspondiendo alrededor de 59\% al sexo masculino. De estos pacientes se efectuaron 1.679 aislamientos. Los aislamientos fueron más frecuentes en sangre $(51,8 \%)$ y en LCR $(27,2 \%)$. Las localizaciones topográficas más frecuentes fueron neumonías y meningitis. La resistencia global de las cepas a penicilina fue de $24,9 \%$, siendo la mayor en México con $47,3 \%$. Argentina y Chile mostraron en ese estu- dio niveles de resistencia del 25 y $28 \%$ respectivamente. Cabe mencionar que los niños bajo dos años de edad presentaron con mayor frecuencia cepas resistentes a penicilina que los niños sobre esa edad. ${ }^{6}$

Recientemente Di Fabio presentó una recopilación sobre el estudio de enfermedad invasora neumocóccica en América Latina. Este estudio incluyó centros de Argentina, Brasil, Colombia, Chile, México y Uruguay. Se incorporaron más de 70 hospitales de más de 30 ciudades. El período de estudio abarcó de 1993 hasta julio de 1999, obteniéndose 4.018 aislamientos en niños con menos de cinco años de edad, siendo $68 \%$ menores de dos años de edad. La enfermedad pulmonar representó $43 \%$ de los aislamientos mientras que la meningitis neumocóccica el $39 \%$. De estos aislamientos $28 \%$ fueron resistentes a penicilina, resultando Brasil el país de América Latina con menor resistencia (20\%) y el mayor México con un $47 \%$. Por otra parte, datos encontrados durante el estudio del proyecto SIREVA-Vigía demuestran claramente que la mayor resistencia se encuentra en los grupos de menor edad. También es claro que la mayoría de las cepas aisladas en este estudio, son resistentes a cotrimoxazol, mientras que la resistencia alta a cefalosporinas con CIM $>1 \mathrm{mg} / \mathrm{ml}$ es muy baja ${ }^{8}$.

Del estudio de 326 cepas obtenidas de enfermedades invasoras provocadas por S. pneumoniae, se pudo demostrar que el $80 \%$ de las cepas con alta resistencia a penicilina (CIM igual o mayor de $1 \mu \mathrm{g} / \mathrm{ml}$ ) son similares a dos clones diseminados por diversos países: "el hispano 23F-1"clone A y "el francés 9V-3" clone B. Todas las cepas del tipo clon A fueron resistentes a cotrimoxazol, tetraciclina y cloranfenicol pero sólo escaso número de cepas fueron resistentes a macrólidos. Este clon se observó en todos los países participantes excepto en Brasil, mientras que el clon B se observó en todos los países?.

Los estudios en Argentina revelan que la enfermedad invasora más frecuente provocada por $S$. pneumoniae fue la neumonía con $52,4 \%$ del total, mientras que la meningitis representó el $23,6 \%$ y la septicemia ocurrió en alrededor del $10 \%$ de los 689 aislamientos ${ }^{10}$. Argentina presenta un perfil de resistencia intermedia en comparación con otros países de América Latina siendo la resistencia global a penicilina de 38\% en 1997 en comparación a 1993, año en que fue de $24,4 \%$ con una diferencia significativa, $\mathrm{p}<0.0001^{10}$. Como era de esperar la resistencia alta a penicilina se observó en el grupo de pacientes afectados con neumonía que en los pacientes con meningitis. Por otra parte los factores de riesgo que se encontraron asociados a la resistencia a penicilina fueron: uso de antimicrobianos 
Tabla 2: Streptococcus pneumoniae: Serotipos más frecuentemente aislados en niños en Latinoamérica*

SEROTIPOS (\%)

\begin{tabular}{lrrrrrr}
\hline PAÍS & 14 & 5 & 1 & $6 \mathrm{~A} / 6 \mathrm{~B}$ & $23 \mathrm{~F}$ & $19 \mathrm{~F}$ \\
\hline ARGENTINA & 31 & 12,8 & 10,9 & 9 & 2,1 & 3,1 \\
BRASIL & 26,6 & 9,6 & 13,7 & 14,9 & 3,6 & 3,8 \\
CHILE & 15,7 & 15,7 & 13,6 & 9,6 & 3,5 & 4,0 \\
COLOMBIA & 2,2 & 10,5 & 9,0 & 15,2 & 9,6 & 7,1 \\
MÉJICO & 11,1 & 4,1 & 1,8 & 13 & 16,4 & 7,6 \\
URUGUAY & 38,6 & 15,8 & 7,6 & 8 & 3,2 & 1,9 \\
& & & & & & \\
\hline
\end{tabular}

* Adaptado de Kertesz D. et al CID 1998; 26: 1355-61

dentro de últimos tres meses, edad inferior a dos años y la presencia de neumonía ${ }^{11}$.

Los estudios efectuados en Chile demuestran la importancia que tiene el neumococo como causa de bacteremia bajo tres años de edad. Así se observó durante el período 1998-1999 la presencia de bacteremia por este microorganismo en niños febriles (fiebre rectal de $40^{\circ} \mathrm{C}$ o más) fue de $\sim 64,6$ casos $100.000 /$ año en niños, tanto en el ámbito ambulatorio como aquellos que requirieron internación. ${ }^{4}$ Otro estudio publicado recientemente sobre 945 aislamientos de $S$. pneumoniae, de los cuales $57 \%$ correspondieron a hemocultivos, mientras que $24 \%$ correspondían LCR, se observó una resistencia a penicilina de $29,4 \%$, siendo de $15,3 \%$ en niños menores de 5 años. ${ }^{12}$

De lo anterior se desprende que el impacto que el neumococo tiene en América Latina, y en particular en nuestros países, es importante. Por lo tanto el desarro110 y eventual empleo de vacunas que sean inmunogénicas en lactantes y que brinden una adecuada eficacia, es altamente recomendable.

Para saber la utilidad de las nuevas vacunas conjugadas anti neumocóccicas es necesario recordar, aunque sea brevemente, los serotipos que prevalecen en la región.

\section{Vacunas conjugadas anti neumocóccicas y serotipos}

S. pneumoniae, a diferencia de $H$. influenzae, presenta un número significativos de serotipos que pueden provocar enfermedad en humanos, de los cuales entre 20 y 40 son los más frecuentes. Por otra parte los serotipos no presentan una distribución homogénea e universal sino por el contrario varían de país a país, e incluso pueden ocurrir variaciones en la prevalencia de los distintos serotipos entre distintas regiones de un mismo país; por lo tanto, el primer paso, es mostrar los serotipos que prevalecen en América Latina. El primer trabajo publicado en este aspecto es el de Kertezs et al sobre los serotipos más frecuentemente aislados por país, lo que se observa en la Tabla 2.

Como puede observarse en dicha tabla, Argentina, Brasil, Chile y Uruguay tienen de alguna manera una incidencia de serotipos similar; también Colombia en alguna medida parecería comportarse en forma parecida a los países anteriormente mencionados. En cambio México es más parecido a lo observado en E.U.A. Como puede observarse el serotipo 14 representó en algunos países de Sudamérica alrededor de $27 \%$ mientras que en México fue sólo de $11 \%$.

Además en este estudio se aislaron 61 diferentes serotipos pero 16 de ellos representaron el $87,7 \%$ de los aislamientos. El otro dato llamativo fue que la presencia de algún tipo de resistencia a la penicilina se observó en 26 serotipos pero los serotipos 14 , 23F, 6B, 19A y $19 \mathrm{~V}$ representaron el $87,5 \%$. En Chile I. Heitmann publicó que en su estudio los serotipos más frecuentes fueron: 14, 1, 5, 6B, 7F, 19F, 3, 6A y $23 F^{12}$

Datos adicionales han sido aportados por el estudio del grupo SIREVA. Así recientemente Di Fabio publicó que los siete serotipos capsulares más comunes fueron $14,6 \mathrm{~A}$ y $6 \mathrm{~B}, 5,1,23 \mathrm{~F}, 19 \mathrm{~F}$ y $18 \mathrm{C}$; si bien hay algunas variaciones regionales, parecería que los tipos capsulares 5 y 1 predominan en el Cono Sur. También resulta evidente que los datos de este último autor son similares a los obtenidos por Kerterz en su publicación de 1998. Adicionalmente destaco datos importantes respecto de resistencia a penicilina y su relación con los serotipos; los serotipos 14 , 23F, 4, 6B, 18C, 19F y 9V representarían el $88 \%$ de las cepas resistentes a penicilina en el estudio de los seis países de América Latina que han participado en este estudio.

Teniendo en cuenta lo anterior, mencionaremos brevemente el estado de desarrollo de las vacunas anti neumocóccicas conjugadas que están disponibles y las que están en vías de desarrollo para su utilidad en los próximos años.

El desarrollo de las vacunas conjugadas contra $S$. pneumoniae se basó en el mismo principio empleado en elaborar las vacunas anti $H$. influenzae b. Esto es, se utilizó el polisacárido capsular como antígeno fundamental y se le agregó como "carrier", transportadores tales como toxoide tetánico, diftérico, 
respuesta de anticuerpos no es uniforme y es específica para cada serotipo.

La eficacia contra enfermedad invasora provocada por S. pneumoniae fue de $97,4 \%$ comparado con el grupo control que recibió vacuna anti meningocóccica C conjugada con el mismo carrier. Por otra parte en el seguimiento de los niños vacunados no se observó un aumento de casos de enfermedad invasora provocada por serotipos no incluidos en la vacuna en el seguimiento a tres años.

Otro de los hallazgos publicados recientemente por Eskola et al fue el relacionado de la eficacia de la vacuna conjugada en niños con otitis media aguda (OMA). El estudio se efectúo en Tampere, Finlandia enrolándose en forma randomizada 1.662 niños que recibieron la vacuna a los 2, 4, 6 y 12 meses de edad. El grupo control recibió vacuna anti hepatitis $B$. La reducción de OMA provocada por neumococo (confirmada por cultivo positivo) fue de $34 \%$ y la disminución de episodios provocados por los serotipos incluidos en la vacuna heptavalente conjugada fue de $57 \%$. Los episodios provocados por otros serotipos, no incluidos en la vacuna se incrementaron en un tercio ${ }^{19}$.

Otro dato importante de esta vacuna fue la demostración efectuada por Dagan et al que las vacunas conjugadas disminuyen el estado de portador orofaríngeo comparativamente al placebo, en forma significativa ${ }^{20}$. La portación faríngea de neumococos aumenta con la edad en los niños; sin embargo, en los niños vacunados con vacuna anti neumocóccica conjugada, la portación de los serotipos de $S$. pneumoniae no aumentó con la edad ${ }^{20}$. Este hallazgo es significativo debido al hecho que la colonización orofaríngea por neumococo es un factor de diseminación de la enfermedad y transmisión del organismo, el uso de vacunas anti neumocóccicas conjugadas podría ayudar a controlar la diseminación de esta bacteria en la comunidad. Se requieren mayores estudios para confirmar estos hallazgos en forma definitiva, pero los datos son alentadores.

\section{Proyección de la vacuna conjugada anti neumocóccica heptavalente en Latinoamérica}

Es evidente que la incorporación de vacunas que protejan contra $S$. pneumoniae es tremendamente importante en el campo de la Pediatría; en este sentido esta vacuna conjugada que contiene 7 serotipos es una herramienta muy útil para el clínico pediatra.

En América Latina, esta vacuna cubriría 58\% de los serotipos que provocan enfermedad invasora neumocóccica, de acuerdo con los datos recientemente publicados por el estudio SIREVA, entre otras razones porque la vacuna no contiene los serotipos 1 y 5 que representan alrededor del $23 \%$ de las enfermedades invasoras. Sin embargo, otro hecho importante es que la cobertura se extendería a $89 \%$ de las cepas de $S$. pneumoniae que provocan enfermedad invasora y que presentan algún tipo de resistencia a penicilina. Este último dato no es menor dado que una de las mayores dificultades en el manejo de las infecciones sistémicas por neumococo son aquellas cepas penicilina-resistentes.

En Argentina la cobertura de la vacuna conjugada de siete serotipos cubriría aproximadamente $57 \%$ de las formas invasoras, con una cobertura del $94,6 \%$ para los serotipos que son resistentes a penicilina. En cuanto a los serotipos que provocan OMA, recientemente finalizó un trabajo efectuado por nuestro grupo demostrando que la vacuna cubriría el 69,8\% de los serotipos de neumococo aislados de oído medio y al $98 \%$ de aquellos que presentan resistencia a penicilina (datos no publicados).

En cuanto a Chile, la vacuna heptavalente conjugada presentaría una cobertura alrededor del 50\% según los datos publicados antes mencionados.

Cabe mencionar que algunos autores plantean la posibilidad que pudiera ocurrir protección cruzadas entre distinto serotipos pertenecientes a un mismo serogrupo $^{20}$, esto esta ampliamente aceptado para el serogrupo 6 donde el 6 A/ 6B presentan protección cruzada. Otros autores consideran que una situación similar podría ocurrir con el serogrupo 19 pero los estudios no son tan categóricos como con el grupo 6. Si la protección inmunológica fuera demostrada en otros serogrupos se podría lograr una protección aún mayor que la aceptada hoy en día.

Es evidente que esta vacuna conjugada de siete serotipos no podría ser incluida en nuestros países dentro de un Programa Nacional de Vacunación dado que se requiere la presencia de un número mayor de serotipos para lograr una cobertura satisfactoria. Las futuras vacunas conjugadas nonavalentes que incluyen los serotipos 1 y 5 nos darían en América Latina coberturas que oscilarían entre 75 al $82 \%$ según los países.

No obstante ello, consideramos que esta vacuna heptavalente puede ser muy útil con un criterio de uso individual, según el criterio del pediatra de cabecera del niño, con el objetivo de evitar enfermedad grave por S. pneumoniae o incluso, disminuir la incidencia de una enfermedad común como es la OMA, especialmente en lactantes. Por otra parte hay un 
grupo de niños bajo dos años de edad que deberían recibir esta vacuna, son aquellos en los cuales la enfermedad neumocóccica invasora provoca una significativa morbimortalidad, tales como: esplenectomizados, niños infectados con el virus de inmunodeficiencia humana, pacientes con enfermedad pulmonar crónica, fibrosis quística, displasia pulmonar, etc. También los cardiópatas severos, diabéticos graves y pacientes con distintos tipos de inmunodeficiencias deberían recibir esta vacuna.

Estamos frente a una vacuna que ofrece una nueva oportunidad para evitar enfermedades graves en nuestros niños, su uso es útil aunque al mismo tiempo presenta las limitaciones propias que siempre ofrece el campo de la Medicina y en particular, el de la Pediatría.

\section{BIBLIOGRAFÍA}

1. MC CRACKEN G H. Etiology and treatment of pneumoniae: Pediatr Infect Dis J 2000; 19: 373-7.

2. PELTOLA H. Haemophilus influenzae type $\mathrm{b}$ disease and vaccination in Latin America and the Caribbean: Pediatr Infect Dis J 1997; 16: 780-7.

3. CATTANEO M, DEVOTO S, VÁZQUEZ M, LÓPEZ E L. Pneumococcal meningitis in children. Abstract 56011. $8^{\text {th }}$ ISID, Boston - Massachussets.

4. LAGOS R. Prospective population-based surveillance for ambulatory invasive pneumococcal disease in children in Santiago, Chile. The Second International Symposium on Pneumococci and Pneumococcal Diseases. Final Programme and Abstract Book. March 19-23, 2000. Abstract \#O26.

5. ORLANDO N, VÁZQUEZ M, MEREDIZ A, TORRADO L, PROCOPIO A, LÓPEZ E L. Prevalence and antibiotic susceptibility of organisms isolated from acute otitis media in Argentinian children. Abstract \# 76.020 . $9^{\text {th }}$ International Congress on Infectious Diseases. Buenos Aires, Arg. April 10-13, 2000.

6. KERTESZ D A, DI FABIO J L, BRANDILEONE M C, et al. Invasive Streptococcus pneumoniae infection in Latin American children: results of the Pan American Health Organization surveillance study. Clin Infect Dis 1999; 26:1355-1361.

7. DI FABIO J L. Streptococcus pneumoniae invasive Disease in Children: Six Years of the Latin American Surveillance Network. Abstract \#10.002. $9^{\text {th }}$ International Congress on Infectious Diseases. Buenos Aires, Arg. April 10-13, 2000.

8. HORTAL M, RUVINSKY R. Surveillance of Invasive Streptococcus pneumoniae Susceptibility to Antimicrobials and Clinical Management of Pediatric Cases: SIREVA-Vigía. Abstract \#10.003. $9^{\text {th }}$ International Congress on Infectious Diseases. Buenos Aires, Arg. April 10-13, 2000.
9. CORSO A, BRANDILEONE MCC. Molecular Epidemiology of Penicillin Resistant S. pneumoniae (PR-SPN) Isolates From Latin-America. Abstract \#10.004 $.9^{\text {th }}$ International Congress on Infectious Diseases. Buenos Aires, Arg. April 10-13, 2000.

10. PACE J, RUVINSKY R, REGUEIRA M, et al. Streptococcus pneumoniae : surveillance in Argentinian children. International Symposium on Pneumococci and Pneumococcal Diseases Abstract Book, 1998; Abstract \#P98.

11. ROSSI A, CORSO A, PACE J, et al. Penicillin-resistant Streptococcus pneumoniae in Argentina: frecuent occurrence of an internationally spread serotype 14 clone. Microb Drug Resist, 1998; 4 (3):225-231.

12. HEITMANN I. Nota breve: Epidemiología nacional de las infecciones causadas por Streptococcus pneumoniae. Rev Chil Infect 1999; 16 (2): 133-136.

13. AHMAN H, KAYHTY H, TAMMINEN P, VUORELA A, MALINOSKI F, ESKOLA J. Pentavalent pneumococcal oligosaccharide conjugate vaccine PncCRM is well-tolerated and able to induce an antibody response in infants. Pediatr Infect Dis J 1996; 15(2): 134-9

14. BLACK S, SHINEFIELD H, FIREMAN B, LEWIS E, RAY $\mathrm{P}$, et al. Efficacy, safety and immunogenicity of heptavalent pneumococcal conjugate vaccine in children: Pediatr Infect Dis J 2000; 19:187-95.

15. ANDERSON E L, KENNEDY D J, GELDMACHER K M, DONNELLY J, MENDELMAN PM. Immunogenicity of heptavalent pneumococcal conjugate vaccine in infants. $\mathrm{J}$ Pediatr 1996; 128:649-53.

16. KING J C, VINK P E, CHANG I, et al. Antibody titers eight months after three doses of a five-valent pneumococcal conjugate vaccine in HIV and non-HIV-infected children less than two years of age. Vaccine 1998;16:361-5.

17. SALADINO R A, STACK A M, MALLEY R, THOMPSON C M, SIVER C R, FLEISER G O. Bacterial polysaccharide immune globulin (BPIG) protects infant rats with $S$. pneumoniae (SP) pneumonia from bacteremia, meningitis and death. Pediatr Res 1996;36:184 ${ }^{\mathrm{a}}$.

18. ESKOLA J, ANTTILA M. Pneumococcal conjugate vaccines. Pediatr Infect Dis J 1999;18:543-51.

19. ESKOLA J, KILPI T, PALMU A, et al.: Efficacy of a pneumococcal conjugate vaccine against acute otitis media: N Engl J Med 2001; 344 (6), Feb. 8: 403-9.

20. DAGAN R, MELAMED R, MUALLEM M, et al. Reduction of nasopharyngeal carriage of pneumococci during the second year of life by a heptavalent conjugate pneumococcal vaccine. J Infect Dis 1996;174:1271-8.

Correspondencia a:

Eduardo López

e-mail: eduardolopez@sinectis.com.ar 